Document downloaded from:

http://hdl.handle.net/10251/139923

This paper must be cited as:

Flores Pedauye, R.; Gago Zachert, SP.; Serra Alfonso, P.; Sanjuan Verdeguer, R.; Elena Fito, SF. (2014). Viroids: survivors from the RNA world?. Annual Review of Microbiology. 68:395-414. https://doi.org/10.1146/annurev-micro-091313-103416

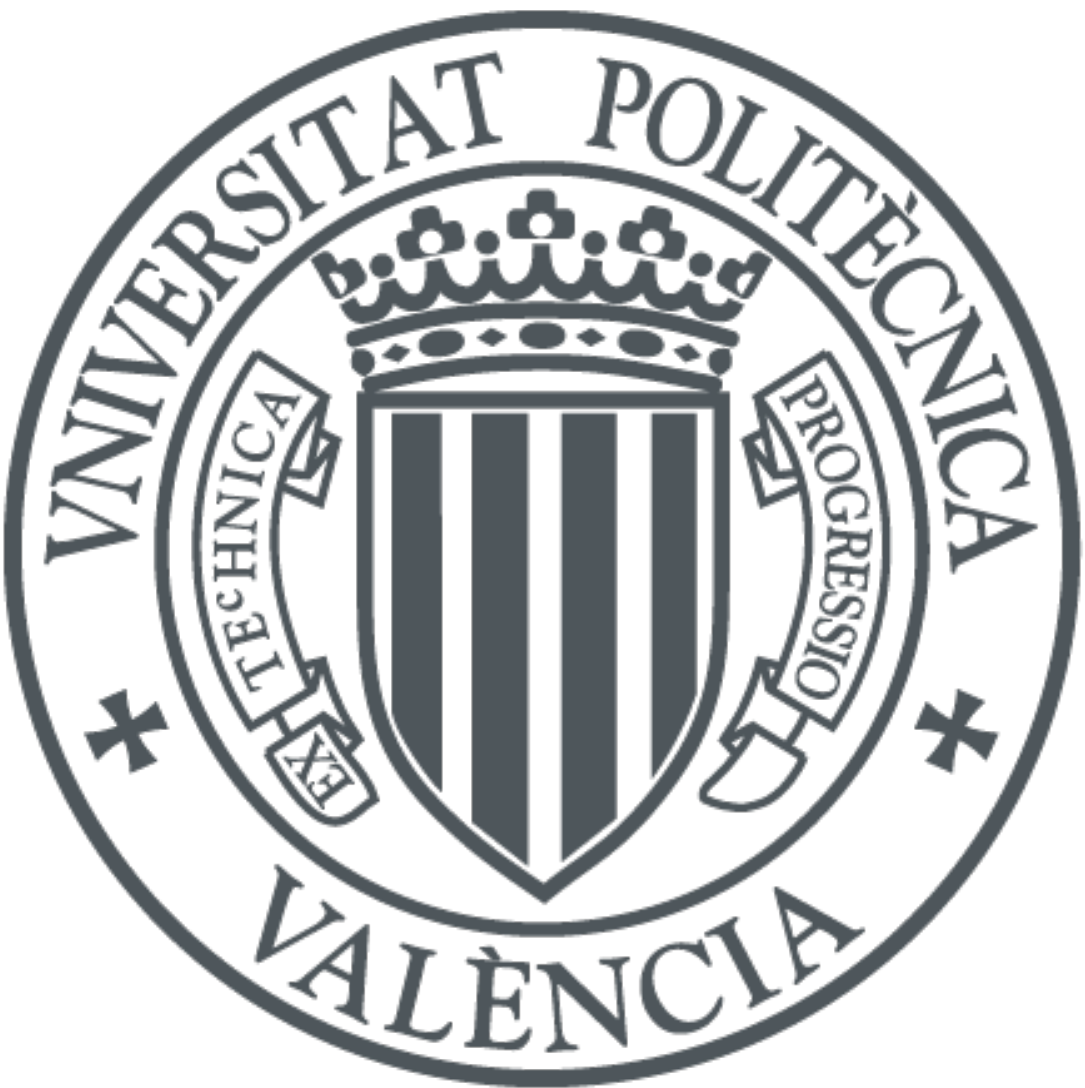

The final publication is available at

https://doi.org/10.1146/annurev-micro-091313-103416

Copyright Annual Reviews

Additional Information 


\section{Viroids: Survivors from the RNA World?}

Ricardo Flores ${ }^{1}$, Selma Gago-Zachert ${ }^{1,2}$, Pedro Serra ${ }^{1}$, Rafael Sanjuán ${ }^{3}$ and Santiago F. Elena ${ }^{1,4}$

${ }^{1}$ Instituto de Biología Molecular y Celular de Plantas (UPV-CSIC), 46022 Valencia, Spain; email: rflores@ibmcp.upv.es

${ }^{2}$ Department of Molecular Signal Processing, Leibniz Institute of Plant Biochemistry, 06120 Halle (Saale), Germany

${ }^{3}$ Instituto Cavanilles de Biodiversidad y Biología Evolutiva, Universitat de València, 46980 Valencia, Spain

${ }^{4}$ The Santa Fe Institute, Santa Fe NM 87501, USA

\section{Keywords}

viroid-like satellite RNAs, hepatitis delta virus, catalytic RNAs, hammerhead ribozymes, small non-coding RNAs

\section{Abstract}

Because RNA can be a carrier of genetic information and a biocatalyst, there is a consensus that it emerged before DNA and proteins, which eventually assumed these roles and relegated RNA to intermediate functions. If such scenario - the socalled RNA World - existed, we may hope to find its relics in our present world. The properties of viroids that make them candidates for being survivors of the RNA World include those expected for primitive RNA replicons: i) small size imposed by error-prone replication, ii) high $\mathrm{G}+\mathrm{C}$ content to increase replication fidelity, iii) circular structure for assuring complete replication without genomic tags, iv) structural periodicity for modular assembly into enlarged genomes, v) lack of protein-coding ability consistent with a ribosome-free habitat, and vi) replication mediated in some of them by ribozymes, the fingerprint of the RNA World. With the advent of DNA/protein life, those proto-viroids lost some abilities and became the plant parasites we now know.

\section{Contents}

INTRODUCTION: WHY THE NEED OF AN RNA WORLD? VIROIDS: ESSENTIAL FEATURES

Discovery of a Subviral World 
Structure: Small Circular RNAs with Compact Folding

Replication: Rolling-Circle Mechanism Catalyzed by Enzymes and Ribozymes

Sequence Diversity

VIROID-RELATED REPLICONS

Viroid-like Satellite RNAs

Retroviroid-like Elements

Hepatitis delta virus (HDV)

WHY VIROIDS (AND VIROID-RELATED REPLICONS) ARE REGARDED AS SURVIVORS OF THE RNA WORLD?

Early Speculations on the Origin of Viroids

Circular RNAs: Relics of Precellular Evolution?

Transition from the RNA World to a World with DNA and Proteins: Viroids

Transition from the RNA World to a World with DNA and Proteins: Viroidrelated Replicons

SUMMARY POINTS 


\section{INTRODUCTION: WHY THE NEED OF AN RNA WORLD?}

RNA has been long considered to play a subsidiary role, serving merely as a link between DNA and proteins, which in all cells store and express the genetic information, respectively. However, this is not the case in the subcelular world, because the genetic material of many viruses - including Tobacco mosaic virus (TMV), the first discovered (Beijerinck, 1898) (4) - is RNA (Fraenkel-Conrat, 1956; Gierer and Schramm, 1956) $(48,54)$. Although replication of some RNA bacteriophages was initially considered to proceed through a mechanism different from that proposed for DNA (Haruna and Spiegelman, 1966) (67), ensuing work with phage Q $\beta$ demonstrated that its single-stranded RNA (ssRNA) of about 4200 nucleotides (nt) replicates by synthezising a complementary strand via base pairing between $\mathrm{A}$ and $\mathrm{U}$ (instead of T as in DNA), and G and C (Feix et al., 1968) (41). Therefore, in these very simple (and presumably very old) self-replicating entities, RNA supplants DNA as carrier of the genetic information. Subsequent discovery of ribozymes, i.e. that some RNAs have catalytic activity (Guerrier-Takada et al., 1983; Kruger et al., 1982) (60, 79), showed that RNA can also express this information, supporting an early proposal that RNA could be the primordial macromolecule (Crick, 1968; Orgel, 1968; Woese, 1967) $(18,100,142)$. This proposal was based in the ablity of ssRNA to fold into complex structures - hairpins with stems stabilized by hydrogen bonds between bases, being extruded as loops the bases with no partner availableresembling that of proteins (Fresco et al., 1960) (50), as well as on its $2^{\prime}$ hydroxyl group providing additional chemical versatility.

The term "RNA World" was coined in a commentary following discovery of ribozymes (Gilbert, 1986) (55): "If there are ribozymes that can catalyze the synthesis of a new RNA molecule from precursors and an RNA template, then there is no need for protein at the beginning of evolution. One can contemplate an RNA world, containing only RNA molecules that serve to catalyze the synthesis of themselves. ... At the next stage RNA molecules began to synthesize proteins, first by developing RNA adapter molecules that can bind activated amino acids, and then by arranging them according to an RNA template using other RNA molecules such as the RNA core of the ribosome. This process would make the first proteins, which would simply be better enzymes than their RNA conterparts. ... Finally, DNA appeared on the scene, the ultimate holder of information copied from the genetic RNA molecules by reverse transcription. After double-stranded DNA (dsDNA) evolved there exists a 
stable linear information store, error correcting because of its double-stranded structure but still capable of mutation and recombination. RNA is then relegated to the intermediary role that it has today - no longer the centre of the stage, displaced by DNA and the more efficient protein enzymes". This enlightening proposal has been extended into an active research field (Atkins et al., 2011) (3). Implicit in the RNA World idea is the existence of primitive RNA replicons of minimal size that later evolved into more complex self-replicating systems. Are in our present-day world remnants of those minimal replicons? In the following sections we will provide evidence supporting that viroids (and some viroid-related replicons) are excellent candidates for being survivors from the RNA World. But we will firstly summarize the unique properties of these genetic elements pertinent to the present context. In-depth reviews dealing with different aspects of viroids have been published previously (Diener, 2003; Ding, 2009; Ding, 2010; Flores et al., 2005; Flores et al., 2011; Flores et al., 2012; Hammann and Steger, 2012; Navarro et al., 2012a; Tabler and Tsagris, 2004; Tsagris et al., 2008) (30, 31, 32, 43, 44, 45, 65, 95, 125, 129).

\section{VIROIDS: ESSENTIAL FEATURES}

\section{Discovery of a Subviral World}

A recurrent situation in science is that unanticipated discoveries entail unanticipated consequences going beyond the initial scheme. Viroids were discovered when attempting to identify the agent of potato spindle tuber (PST) disease, suspected to be a virus. The finding that the PST agent could be transmitted to tomato, easier to grow and expressing symptoms in a shorter time, expedited the experiments and produced the first surprise: after ultracentrifugation of tomato extracts, the infectious principle remained in the supernantant instead of associated with the presumed viral particles - the virions, characteristically formed by protein subunits encapsidating the nucleic acid - expected to accumulate in the sediment. Besides, this infectious principle moved in sucrose gradient centrifugation much slowly than typical virions, and its mobility remained unchanged after deproteinating treatments, indicating that the PST agent might be a naked nucleic acid. This hypothesis was tested with polyacrylamide gel electrophoresis (PAGE): the PST infectious principle migrated as a band, absent in mock-inoculated plants, with the mobility predicted for a minuscule nucleic acid and, more explicitly, for an RNA (because of its sensitivity to RNase, but not to DNase). At this stage the term viroid was proposed for what 
appeared the first autonomously-replicating subviral agent (Potato spindle tuber viroid, PSTVd) (Diener, 1971; Diener, 1972) (26, 27). Application of this methodology quikly resulted in discovery of novel viroids as the causal agents of plant diseases of presumed viral etiology, but for which the expected virions had not been identified. Altogether these studies opened a window onto a subviral world composed of small self-replicating RNAs, overthrowning viruses from the lowest step of the biological scale that they had occupied along the first 65-70 years of the $X X^{\text {th }}$ century. It is worth noting that the subviral world, like the viral world, was discovered from studies on plant systems. However, in contrast to viruses, viroids are restricted to the plant kingdom so far, although at least one viroid-related replicon is involved in a human disease (see below).

\section{Structure: Small Circular RNAs with Compact Folding}

First of all, how small was PSTVd compared with the genomic RNAs of known viruses? Early estimations inferred from PAGE, corroborated by electron microscopy of purified PSTVd RNA (Sogo et al., 1973) (123), resulted in about 300 nt, a size at least 10-fold smaller than that of the ssRNAs of TMV (6400 nt) and phage Q $\beta$ (see above). Moreover, electron microscopy of denatured PSTVd RNA produced a second surprise: the RNA was circular, a feature previously conjectured given its resistance to exonucleases. Together, the small size (corresponding to potential translation products of minimal complexity) and circularity (hampering translation by conventional ribosome scanning from a $5^{\prime}$ terminus), suggested that PSTVd was a non-protein-coding RNA. Determination of its 359-nt primary structure by direct RNA sequencing (a milestone in Molecular Biology since it was the first eukaryotic pathogen completely sequenced) confirmed the circular structure and the absence of typical initiation codons (Gross et al., 1978) (58). From a functional perspective this was a distinctive point, because all viruses encode proteins that mediate one or more steps of their biological cycle. Furthermore, thermodynamic predictions (Riesner et al., 1979) (108), along with in vitro probing with RNases and bisulphite, supported intramolecular folding of PSTVd into a rod-like secondary structure -also favored by circularity and composed by double-stranded segments flanked by apparently unstructured loops- with a width akin to that of a dsDNA as observed by electron microscopy under non-denaturing conditions.

Sequencing of other viroids confirmed preservation of the rod-like folding and, within it, of a central conserved region (CCR) (McInnes and Symons, 1991) (89), 
which emerged as an idiosyncratic trait (Fig. 1: viroid structure). However, this was not the case of Avocado sunblotch viroid (ASBVd) (Symons, 1981) (125), without a CCR but with their strands of both polarities displaying a remarkable behavior: they selfcleaved through hammerhead ribozymes, thus acting as catalytic RNAs (Hutchins et al., 1986) (73), an attribute with deep implications in its replication and evolutionary origin. PSTVd and ASBVd epitomized two viroid groups, which ultimately resulted in the families Pospiviroidae y Avsunviroidae respectively (Elena et al., 1991; Flores et al., 2005) $(36,43)$, a cataloging consistent with other distinctive features that include the subcellular site and mode of replication (see below) (Fig 2: viroid families). Moreover, some members of the Avsunviroidae, like Peach latent mosaic viroid (PLMVd) (Hernández and Flores, 1992) (69) and Chrysanthemum chlorotic mottle viroid (CChMVd) (Navarro and Flores, 1997) (92), fold into branched secondary structures (Fig. 1: viroid structure).

\section{Replication: Rolling-Circle Mechanism Catalyzed by Enzymes and Ribozymes}

Because viroids are non-protein-coding RNAs, they need for their replication to usurp the transcription and processing machinery of their hosts. In other words, viroids are essentially transcriptional parasites, while viruses are essentially translational parasites (since for achieving infection they need to express the proteins they encode). Viroid replication takes place in the nucleus (family Pospiviroidae) or in plastids, mostly chloroplasts (family Avsunviroidae), through a rolling-circle mechanism with RNA intermediates (Branch and Robertson, 1984; Daròs et al., 1994; Grill and Semancik, 1978; Hutchins et al., 1985) $(7,19,57,72)$. The infecting circular (+) RNA (the sign is allocated conventionally to the most abundant strand in vivo) is repeatedly transcribed into oligomeric (-) strands. These replicative intermediates, by themselves (asymmetric pathway) or after being cleaved and ligated into circular monomers (symmetric pathway), serve as template for synthesis of oligomeric $(+)$ strands that are finally processed into their monomeric circular counterparts (Fig. 3: rolling-circle mechanism).

Therefore, three catalytic activities are required: RNA polymerase, RNase, and RNA ligase, the characterization of which have led to unexpected discoveries. First, despite the existence of several RNA-dependent RNA polymerases (RDRPs) in plants (Schiebel et al., 1998; Wassenegger and Krczal, 2006), transcription of viroid strands is catalyzed by nuclear or plastidic DNA-dependent RNA polymerases (DDRPs) forced to accept RNA templates (Flores and Semancik, 1982; Mühlbach and Sänger, 
1979; Navarro et al., 2000; Rodio et al., 2007; Schindler and Mühlbach, 1992) (42, 91, 93, 112, 118). Second, cleavage of oligomeric strands is mediated in the family Pospiviroidae by a class-III RNase enzyme that operates on a dsRNA structure formed by the upper CCR strand of two consecutive units (Gas et al., 2007) (53), or by cisacting hammerhead ribozymes in the family Avsunviroidae. And third, circularization occurs by a plastidic isoform of the tRNA ligase (Nohales et al., 2012a) (97) or, remarkably, by nuclear DNA ligase I (redirected to circularize RNA substrates) (Nohales et al., 2012b) (98). Therefore, as a manifestation of their extraordinary parasitic abilities, viroids reprogram the template specificity of a DDRP to function as an RDRP, and the substrate specificity of a DNA ligase to operate as an RNA ligase. To complete their infectious cycle, viroids need additionally to move for invading distal plant parts. Recent data indicate that specific loops/bulges in the rodlike structure of PSTVd and related viroids, instead of unstructured, are stabilized by arrays of non-canonical pairs, with some of these elements playing a role in replication and others in systemic trafficking (Ding, 2009; Ding, 2010; Zhong et al., 2008) $(31,32,146)$.

\section{Sequence Diversity}

As a consequence of their high mutation rates (see below), some viroids display high genetic variability. This phenomenon has been observed after inoculating plants with infectious viroid-cDNAs (usually head-to-tail dimeric constructs) or their transcripts (mimicking replicative intermediates), and quantifying the heterogeneity of the resulting progeny. The diversity observed in the family Pospiviroidae, e.g. PSTVd (Góra-Sochacka et al., 1997) (56), Citrus exocortis viroid (Bernad et al., 2009; Hajeri et al., 2011; Visvader and Symons, 1985) (6, 63, 134), Citrus bent leaf viroid (Gandía and Duran-Vila, 2004) (52), Chrysanthemum stunt viroid (CSVd) (Codoñer et al., 2006) (16), and Citrus dwarfing viroid (Tessitori et al., 2013) (130), is relatively low and the consensus sequence does not deviate significantly from the wild-type. However, in the family Avsunviroidae, e.g. PLMVd (Ambrós et al., 1999; Navarro et al., 2012) (2, 96) and CChMVd (Codoñer et al., 2006; De la Peña et al., 1999) (16, 22), the resulting population is a complex spectrum of mutants and the consensus sequence often changes over time or is difficult to define. This difference is most likely caused by the distinct replication fidelity of the RNA polymerases involved (see below), with selection imposed by the host also shaping the final distribution of genetic variants 
observed (Bernad et al., 2009; Semancik et al., 1993; Tessitori et al., 2013; Wassenegger et al., 1996) $(5,121,130,137)$.

Given their small genomes and high mutation rates, viroids have been often treated in the framework of the quasispecies theory, originally developped to describe natural selection acting on primitive RNA replicons undergoing error-prone replication and consisting of mutant distributions (mutant spectra or mutant clouds) (Biebricher and Eigen, 2006; Eigen, 1971) $(6,34)$. This theoretical scheme was first applied to phage $Q \beta$ (Domingo et al., 1978) (33) and then to many other RNA viruses (Lauring and Andino, 2010; Ojosnegros et al., 2011; Vignuzzi et al., 2006) (83, 99, 133). A genuine prediction of the quasispecies theory that discriminates it from classic population genetics models, is that selection operates on sets of variants from a particular region of the fitness landscape rather than on individual virus variants. As a result, the fitness associated with a specific sequence depends on the average fitness of its neighbors in the sequence space (Lauring and Andino, 2010) (83), as first experimentally shown with the RNA phage $\phi 6$ (Burch and Chao, 1999) (11). This collective behaviour can lead to the so-called "survival of the flattest" effect, whereby a population located in a region of the fitness landscape where a large proportion of sequence neighbors are selectively neutral can outcompete another population located in a higher-fitness peak but with a more deleterious sequence neighborhood (Schuster and Swetina, 1988; Wilke et al., 2001) (120, 140). This effect was first demonstrated in vivo by competition experiments between CSVd and CChMVd, performed at different conditions: at physiological mutation rate the fittest CSVd outcompeted CChMVd, but increasing mutation rate by UV irradiation reverted the fortune and the flattest CChMVd outcompeted CSVd (Codoñer et al., 2006) (16). Later on, the survival of the flattest was also shown in Vesicular stomatitis virus populations subjected to increased mutational stress by chemical mutagenesis (Sanjuán et al., 2007) (116).

Recent work has suggested that interactions such as genetic complementation and interference among individual components of the viral population also determine to a good extent its behavior, with interference most likely being exerted by defective gene products resulting in non-functional protein complexes during the virus biological cycle (Ojosnegros et al., 2011) (99). However, since viroids are non-proteincoding RNAs, complementation by protein products acting in trans cannot exist and interference must occur at the RNA level. 


\section{VIROID-RELATED REPLICONS}

\section{Viroid-like Satellite RNAs}

Besides viroids, three other viroid-related subviral elements deserve mention in the context of the RNA World. Plant viroid-like satellite RNAs are structurally similar to viroids, being just composed by a small non-protein-coding circular RNA (Schneider, 1969; Roossinck et al., 1992; Symons and Randles, 1999) (119, 113, 126). Additional similarities include functional aspects, because viroid-like satellite RNAs replicate through a rolling-circle mechanism with ribozyme-mediated self-cleavage of the resulting RNA oligomeric strands of one or both polarities. However, ribozymes are not only of the hammerhead class (Forster and Symons, 1987; Prody et al., 1986) (47, 105), but also of the hairpin class (Buzayan et al., 1986) (12). More importantly, replication of viroid-like satellite RNAs is not autonomous, but relying on the RNA replicase complex encoded —at least in part- by a co-infecting helper RNA virus (by whose coat protein are encapsidated for transmission); the term satellite refers to this dual dependence (Bruening et al., 1991) (10). Because the helper RNA viruses replicate in organelle-associated membranous vesicles connected with the cytoplasm (den Boon and Ahlquist, 2010) (25), replication of viroid-like satellite RNAs most likely also takes place in these vesicles, although the lack of direct supporting data leaves open the possibility of the helper virus encoding protein(s) that redirect a host RNA polymerase for this purpose (Symons and Randles, 1999) (126). Resembling the situation found in the family Avsunviroidae, circularization of unit-length strands resulting from self-cleavage in viroid-like satellite RNAs might be mediated by a cytoplasmic isoform of the tRNA ligase, or by self-ligation (the RNA ligase activity of the hairpin ribozyme is higher than that of the hammerhead ribozyme) (Buzayan et al., 1986; Fedor, 2000) (12, 40).

\section{Retroviroid-like Elements}

There is a second class of plant small circular RNA similar to viroid and viroid-like RNAs in size and in harboring hammerhead ribozymes (Hernández et al., 1992) (70), but with a singular property: the RNA is accompanyied by a homologous DNA counterpart fused to sequences of either a pararetrovirus or the host (Daròs and Flores, 1995; Hegedus et al., 2004) (20, 68). This class, dubbed retroviroid-like elements because the homologous DNA is presumably generated by a reverse transcriptase $(\mathrm{RT})$ - pararetroviruses encode such an enzyme - is restricted so far to 
some carnation sources and it cannot be transmitted horizontally. The presence of hammerhead ribozymes in both polarity strands of this carnation circular RNA supports its replication through a symmetric rolling-circle mechanism, but the lack of horizontal transmission suggests that a supply of transcripts from the DNA form is needed.

\section{Hepatitis delta virus (HDV)}

In contrast with the other two, the third class of viroid-related subviral entities has been reported in animals, specifically in humans. With an approximate size of 1680 nt, the RNA of HDV - the smallest genome of an animal virus - occupies in the biological scale of genomic sizes the position immediately above viroids, with which it displays striking structural similarities: circularity and folding into a rod-like secondary structure (Chen et al., 1986; Rizzeto et al., 1977; Rizzeto et al., 1980; Wang et al., 1986) (14, 109, 110, 135). Extending these similarities, HDV RNA replicates autonomously in the nucleus through a rolling-circle mechanism catalyzed by host enzymes (an RNA polymerase redirected to transcribe RNA templates and, presumably, an RNA ligase), and by cis-acting ribozymes idiosyncratic to this infectious agent (Kuo et al., 1988a; Kuo et al., 1988b) (81, 82). However, HDV RNA encodes in its antigenomic polarity a protein (like some RNA viruses) (Wang et al., 1986) (135), and depends for transmission on a helper virus, Hepatitis B virus (like viroid-like satellite RNAs).

\section{WHY VIROIDS AND VIROID-RELATED REPLICONS ARE REGARDED AS SURVIVORS OF THE RNA WORLD?}

\section{Early Speculations on the Origin of Viroids}

Initial ideas suggested that viroids could be "escaped introns", thus descending from host RNAs. This proposal was based on: i) sequence similarities of PSTVd (and other viroids) with group I and II introns, and ii) specific cleavage of viroid oligomeric replication intermediates and their following ligation into circular forms, conceptually resembling cleavage-ligation by which introns are self-spliced (and circularized) of primary transcripts and exons are joined into mature RNAs (Diener, 1981; Hadidi, 1986) $(28,62)$. Yet, the subsequent finding that the reaction mechanisms were distinct eroded the credibility of the viroid-intron connection. 
On the other hand, comparative sequence analysis of PSTVd and two related viroids unvealed conspicuous similarities with the ends of transposable elements, which together with the presence of inverted repeats and flanking imperfect direct repeats, suggested that viroids may have originated from transposable elements or retroviral proviruses by deletion of internal fragments (Kiefer et al., 1983) (78). However, despite initial reports indicating the presence of PSTVd sequences in cellular DNA of normal plants, further analyses failed to confirm these results and, moreover, ASBVd (and later other members of its family) did not fit this model. Therefore, viroids do not seem to be simplified versions of viruses or transposons.

\section{Circular RNAs: Relics of Precellular Evolution?}

With this provoking title Diener (1989) (29), gave a twist to previous perspectives and proposed that viroids and viroid-like satellite RNAs were more likely candidates than introns as "living fossils" of a precellular RNA World — precellular should be understood lacking cells as we know them today- and showed that their characteristic features might have evolved to cope with barriers in the self-replication of primitive RNAs. Further studies have reinforced this view and filled some gaps. Hereafter we will discuss the evidence that supports Diener's claim, collecting his arguments and supplying others (Fig. 4: viroids, the oldest replicons?).

First, the intrinsic error-prone replication of primitive RNA systems imposed a limit to the size of their master (predominant) sequences to overcome the "error catastrophe" leading to extinction (Eigen, 1971) (34). Thus, the evolutionary paradigm "the simplest, the oldest", which with some limitations is generally accepted, seems appropriate for viroids because they are the smallest known replicons.

Second, to increase replication fidelity, $(\mathrm{G}+\mathrm{C})$-rich sequences would have been selected in primitive RNA systems due to the higher thermodynamic stability of GC pairs versus their AU counterparts (Eigen and Schuster, 1978) (35). Viroids also meet this requirement: they are $(\mathrm{G}+\mathrm{C})$-rich with a notable exception, ASBVd (Symons, 1981) (125). Recently, it has been proposed that the conflict intrinsic to the RNA World between stable folding (required for ribozyme activity) and template ability (required for replication), could have been solved by a "division of labor" between the two RNA strands, with one (containing wobble G:U pairs) folding into a ribozyme and the reverse complement (containing the less stable C:A mispairs) functioning as genome (Ivica et al., 2013) (74). Analysis of 40 viroid sequences is 
consistent with this proposal, a finding remarkable because they must have evolved in response to different selection pressures beyond a simplified "division of labor" (Ivica et al., 2013) (74). However, this proposal should be restricted to members of the family Avsunviroidae, the only ones with hammerhead ribozymes. Furthermore, since the ribozymes can be formed by the two strands and both function as templates (Fig. 3: rolling-circle mechanism), the "division of labor" view becomes blurred.

Third, circularity circumvents achieving complete replication without recurring to genomic tags, which mark specific initiation sites in linear templates and ensure their end-to-end copying. Even if polymerization of viroid strands in the present cellular habitat starts at defined sites (Delgado et al., 2005; Kolonko et al., 2006; Navarro and Flores, 2000) $(24,79,94)$, this may have not occurred in primitive replicons, for which reiterative copying of their circular genomes -irrespective of initiation at a defined position- would avoid loss of genetic information. Moreover, this rolling-circle replication would result in multiple copies of the genetic information, a condition likely favored in primitive replicons with a high mutation rate (Reanney, 1984) (106). Inherent to this replication mode is the need for a processing mechanism (cleavage and circularization) of the oligomeric replication intermediates (Fig. 3: rolling-circle mechanism). Members of the family Avsunviroidae fulfill the first step of this mechanism, because their oligomeric strands self-cleave through hammerhead ribozymes; the second step appears also feasible, as revealed by the high RNA ligase activity associated with the hairpin ribozymes present in some viroid-like satellite RNAs (Bruening et al., 1991; Buzayan et al., 1986) $(10,12)$.

Fourth, the sequence of some viroids exhibits a structural periodicity characterized by repeat units of different length. Although this periodicity was initially associated to the protein-binding ability of viroids (Juhasz et al., 1988) (77), other interpretations pointed to a mechanism by which larger genomes could have evolved (Diener, 1989) (29). Even if the strength of this argument is weakened by the lack of structural periodicity in at least one viroid and in most viroid-like satellite RNAs (Diener, 1989) (29), the idea that viroids could have been built modularly seems atractive, particularly if several instead of a single module are considered. The recombinant nature of some viroids, which are chimeras formed by fragments present in other viroids, is consistent with this view (Hammond et al., 1989; Rezaian, 1990) (66; 107). Moreover, modular evolution is more advantageous than direct evolution of large functional molecules because of the higher mutation rates allowed, the shortening of evolutionary times, and the possible emergence of complex structures that could not 
be otherwise directly selected (Manrubia and Briones, 2007) (87). According to computational predictions the module repertoire would be dominated by hairpinlike structures, a fraction of which could have RNA ligase activity and catalyze assembly of larger, eventually functional RNAs; this stepwise ligation-based model of modular evolution could ease the path to the appearance of a ribozyme with RNA replicase activity (Briones et al., 2009) (9).

Fifth, viroids are non-protein-coding RNAs. This key feature not only discriminates viruses from viroids, but also traces their putative origin back to what has been called the first age of the RNA World. This first age was delimited between the emergence of the first replicating RNA cell(s) - a metabolism without cellular confinement seems unlikely - and just before the advent of the ribosome, which opened the second age of the RNA World; this second age lasted until the appearance of the first replicating DNA cell(s), which in the Darwinian competition eliminated their parental RNA cell counterparts (Forterre, 2005) (46). Even the catalytic component of present-day ribosomes is RNA, with proteins just providing a scaffold: "the ribosome is a ribozyme", as has been vividly summarized (Cech, 2000) (13). Thus, viroids comply with the lack of mRNA activity expected for a replicating relic of the (first age) RNA World, wherein no ribosomes existed.

And sixth and most important, some viroids display catalytic activity via hammerhead ribozymes. When Diener formulated his hypothesis, the presence of ribozymes (the key signature of the RNA World) was prevalent among viroid-like satellite RNAs, but restricted to just one (and peculiar) viroid (ASBVd). Subsequent discovery of three additional viroids with hammerhead ribozymes in both polarity strands, PLMVd (Hernández and Flores, 1992) (69), CChMVd Navarro and Flores, 1997) (92) and Eggplant latent viroid (Fadda et al., 2003) (39) - and more recently of two other candidates (Wu et al., 2012; Zhang et al., 2013) (143, 145) — dispelled the doubts that ASBVd could be a viroid-like satellite RNA and consolidated the family Avsunviroidae. The small size and relatively low structural complexity of the hammerhead and hairpin ribozymes, much simpler than those mediating intron splicing, also support their involvement in self-replication of RNA. Of the three steps of viroid replication — RNA polymerization, cleavage and ligation (Fig. 3: rollingcircle mechanism) - the hammerhead and hairpin ribozymes can catalyze cleavage (and the second also ligation). RNA-catalyzed RNA polymerization has not been reported in nature but in vitro evolution studies have shown that RNA can catalyze 
this reaction (Johnston et al., 2001; Lincoln and Joyce; 2009; Wochner et al., 2011) (76, $86,141)$, thus making feasible viroid replication in a pre-protein world.

Do viroids have a monophyletic origin? The low overall sequence similarity among these RNAs poses a problem for inferring a reliable phylogeny (Jenkins et al., 2000) (75). Nevertheless, when alignments are adjusted —considering the local similarities and the insertions/deletions and duplications/rearrangements described - and an appropriate estimator of genetic distances is used, phylogenetic reconstructions support a monophyletic origin. Furthermore, these reconstructions are consistent with the major groups proposed previously for viroids (and viroid-like satellite RNAs), as well as with their biological properties (Elena et al., 1991; Elena et al., 2001) (36, 37). Yet, the possibility of a polyphyletic viroid origin cannot be dismised, particularly considering the unsual base composition of ASBVd. On the other hand, thermodynamic predictions of the secondary structure of viroid RNAs support an evolutionary trend during viroid radiation towards increased robustness (resistance to deleterious mutations) and reduced antagonistic epistasis (interactions between deleterious mutations) (Sanjuán et al., 2006a; Sanjuán et al., 2006b) (114, 115).

\section{Transition from the RNA World to a World with DNA and Proteins: Viroids}

Since nearly all intermediate stages have been faded by time, it is difficult to speculate how viroids evolved from the RNA World, more than 3000 million years ago, into a subsequent world involving two additional macromolecules (proteins and DNA). Nevertheless, at least some hints can be inferred from a close examination of these small replicating RNAs. Focusing on their clear discrimination into the families Pospiviroidae (or nuclear viroids) and Avsunviroidae (or chloroplastic viroids), two reasons argue in favor of the latter preceding the former. According to the endosymbiont hypothesis, plastids derive from primitive cyanobacteria by symbiosis (Margulis, 1993) (88); these free-living cells, which emerged prior to eukaryotic cells, might have hosted (and perhaps still host) the ancestors of the family Avsunviroidae before they adapted to plants (Chela-Flores, 1994; Lima et al., 1994) (14, 85). In support of this view, the hammerhead ribozyme mediating one step of the replication cycle has been retained only in the family Avsunviroidae. Conceivably, the most complex nuclear environment provided increased opportunities for the complete transition from RNA- to protein-catalyzed replication, while this transition has been partially accomplished in plastids. Yet, it is intriguing that the two families 
have adopted the same strategy regarding polymerization of RNA strands: to redirect DNA-dependent RNA polymerases to transcribe RNA templates. While such scenario may appear realistic for viroids replicating in plastids, wherein no RNA-dependent RNA polymerase has been described, the situation is different for viroids replicating in the nucleus, wherein several enzymes of this class accumulate. An alternative and more plausible explanation is that the DNA-like compact folding of the genomic viroid RNA (and its replicative intermediates) may have facilitated their recognition by RNA polymerases that transcribe double-stranded templates into ssRNAs, in contrast to RDRPs that recognize ssRNA templates and convert them into dsRNA products.

Of the two DDRPs residing in plastids (Allison et al., 1996; Sugiura, 1992) (1, 124), the available evidence supports the involvement of the nuclear-encoded polymerase (NEP) in replication of members of the family Avsunviroidae (Navarro et al., 2000; Rodio et al., 2007) $(94,112)$. This finding is consistent with the NEP, composed by a single subunit, being structurally simpler (and presumably older) than the plastidencoded polymerase (PEP) composed by five subunits. On the other hand, the three (I to III) nuclear DDRPs typical of eukaryots (Cramer et al., 2008) (17), and the two additional ones (IV and V) described recently in plants (Haag and Pikaard, 2011) (61), display high structural complexity (with more than 10 subunits). Why members of the family Pospiviroidae have specifically co-opted RNA polymerase II (Flores and Semancik, 1982; Mühlbach and Sänger, 1979; Schindler and Mühlbach, 1992) (42, 91, 118) remains an enigma but, interestingly, the same enzyme is also involved in HDV RNA replication (Taylor, 2009) (128). Perhaps this aptitude of RNA polymerase II is related to its versatility in catalyzing transcription of protein-coding genes, as well as long and small non-coding RNAs (Haag and Pikaard, 2011) (61).

The RNA polymerases mediating replication of viroids are also the driving force that largely determines two of their features: the differential complexity of viroid quasi-species and the recombinant nature of some viroids. The mutation rate estimated for CChMVd, 0.0025 per site and replication cycle (one mutation per replicated genome), is the highest reported for any biological entitiy (Fig. 5: mutation rate scale) (Gago et al., 2009) (51). This extremely error-prone replication —most likely caused by a proofreading-deficient RNA polymerase (NEP) forced to use RNA instead of its native DNA template (Flores et al., 2005) (43), and perhaps also by the accumulation of mutagenic free radicals in plastids or unbalanced nucleotide poolsis reminiscent of that postulated for the primitive replicons of the RNA World. 
Viroids, and more explicitly members of the family Avsunviroidae, can possibly withstand this elevated mutation rate because they have a very small genome, whereas more complex genomes would accumulate an excessive mutational load (Eigen, 1971) (34). These results also suggest that emergence of mechanisms enhancing replication fidelity was of key importance for the evolution of complexity in the early history of life (Gago et al., 2009) (51). Furthermore, the need to transcribe atypical viroid RNA templates might also decrease the processivity of the otherwise DDRPs, promoting their stalling and subsequent jumping with the bound nascent transcript, thus facilitating the appearance of chimeric viroids. Their recurrent identification (Verhoeven et al., 2013) (132), supports that recombination has played an important role in shaping the "viroidsphere".

The other two replication steps further attest to the resilience of viroids. For cleavage of their oligomeric replication intermediates, members of the family Pospiviroidae recruit RNases of class III mediating post-transcriptional maturation of highly-structured nuclear transcripts (Gas et al., 2007) (53), and members of the Avsunviroidae recruit RNA-binding proteins with a similar role in processing of chloroplastic transcripts, which additionally behave as RNA chaperones that in vitro, and presumably in vivo, stimulate the efficiency of hammerhead ribozymes (Daròs and Flores, 2002) (21). For circularization of the resulting unit-length strands, PSTVd (and most likely the other members of the family Pospiviroidae) divert DNA ligase I to function as an RNA ligase taking advantage that, upon DNA binding, DNA ligase I promotes a local RNA-like A-form conformation in the vicinity of the DNA break (Pascal et al., 2004; Pascal, 2008) (101, 102). Moreover, DNA ligase I and some RNA ligases, like T4 RNA ligase 1, display the same substrate specificity (5'phosphomonoester and 3'-hydroxyl termini) as those generated by RNases of class III and operate through a similar mechanism (Ho and Shuman, 2002; Wang et al., 2006) (71, 136). Further supporting this view, DNA ligases, RNA ligases, and RNA-capping enzymes probably evolved by fusion between auxiliary effector domains and an ancestral catalytic module mediating RNA repair (Shuman and Lima, 2004) (122). Thus, some viroids would oportunistically exploit the preservation of a primordial role of DNA ligase I (Nohales et al., 2012b) (98). In contrast, for ligation of the (+) and (-) monomeric linear replication intermediates, members of the family Avsunviroidae recruit a chloroplastic tRNA ligase isoform with specificity for the $5^{\prime}$-hydroxyl and $2^{\prime}, 3^{\prime}$ cyclic phosphodiester termini produced by hammerhead ribozymes (Nohales et al., 2012a) (97). 
Transition from the RNA World to a World with DNA and Proteins: Viroid-related Replicons

The features of viroid-like satellite RNAs, both structural (small size and circularity) and functional (lack of mRNA ability and replication by a rolling-circle mechanism mediated by hammerhead and hairpin ribozymes), support a common origin with viroids and, particularly, with members of the family Avsunviroidae (Elena et al., 2001) (37). However, in their subsequent evolution, viroid-like satellite RNAs have followed a different strategy: instead of relying exclusively on the host transcriptional and processing machinery, they have additionally parasitized a helper virus that provides the transmission and most likely part of the replication machinery. This "ménage à trois", more complex but possibly more efficient for ensuring dispersal of the viroid-like RNAs by the helper virus - viroids depend in this respect solely on their own - also suggests that parasitism emerged very early in the history of life.

Resembling viroid-like satellite RNA, HDV has also evolved to parasitize a helper virus but only for transmission because HDV RNA, like viroids, replicates autonomously in the infected cells. How HDV RNA might have appeared has been discussed in extenso previously (Taylor and Pelchat, 2010) (129). Here we will just emphasize that analysis of HDV RNA has uncovered its recombinant nature: the rodlike secondary structure can be regarded as the fusion of a viroid-like domain, holding the ribozymes and located in one of the terminal domains, to another domain that in the antigenomic polarity contains the open reading frame for the socalled delta antigen $(\delta \mathrm{Ag})$, a protein with a crucial role in the biology of the virus (Gudima et al., 2000) (59). This modular desing has led to the proposal that HDV RNA might have arisen by a self-replicating viroid-like RNA capturing the mRNA encoding a protein, like the $\delta \mathrm{Ag}$, which having a beneficial effect on the recombinant product would have secured its persistence (Brazas and Ganem, 1996; Robertson, 1996) $(8,111)$.

In contrast to viroids and the other viroid-related replicons, retroviroid-like elements have a DNA counterpart and, therefore, should have appeared following the "invention" of this biopolymer. DNA can be viewed as a derivative of RNA, since all organisms synthesize deoxyribonucleotides by reducing ribonucleotides, and thymine by methylating uracil (Lazcano et al., 1988) (84). The first of these two reactions, due to its complex sulfur-based mechanism, seems unlikely to have been 
catalyzed by a ribozyme, thus demanding a protein enzyme. This argument and others (Freeland et al., 1999; Lazcano et al., 1988) $(49,84)$, support the view that DNA appeared during the second age of the RNA World (after the advent of the ribosome) (Forterre, 2005) (46). RT, mediating transition from RNA to DNA, most likely evolved from an ancestral RDRP - ribozyme or protein enzyme- involved in replication of early RNA genomes (Moelling, 2013; Poole and Logan, 2005; Xiong and Eickbush, 1990) $(90,103,144)$. By adquiring an homologous DNA from which RNA can be transcribed, retroviroid-like elements would have increased their survival chances. Intriguingly, following initial discovery of hammerhead ribozymes in certain newt transcripts (Epstein and Gall, 1987) (38), recent sudies have unveiled the existence of hammerhead and HDV-like ribozymes along the biological scale, with the insertion site of the corresponding DNAs in the host genomes indicating a possible regulatory function and the contribution of retrotransposition to their genesis (De la Peña and García-Robles 2010; Hammann et al., 2012; Webb et al., 2009) $(23,64,139)$. However, it is unclear whether the RNA products resulting from ribozyme-mediated self-cleavage are subsequently circularized.

\section{SUMMARY POINTS}

"The time had come to ask how the DNA $\rightarrow$ RNA $\rightarrow$ protein flow of information had ever got started. Here, Francis (Crick) was again far ahead of his time. In 1968 he argued that RNA must have been the first genetic molecule, further suggesting that RNA, besides acting as a template, might also act as an enzyme and, in so doing, catalyze its own self-replication". The course of time has provided accumulating evidence to this passionate laudation by J. D. Watson in the prologue of the first edition (1993) of the classic book on the RNA World (Atkins et al., 2011) (3). Just a recent example: the synthesis of activated pyrimidine ribonucleotides starting from plausible prebiotic precursors and conditions, has bypassed the difficulty of how ribonucleotides could have been formed from their constituent parts (ribose and nucleobases), and removed another important hurdle for the emergence of the RNA World (Powner et al., 2009) (104). The survival until today of viroids (and viroidrelated replicons) adds further support to this view.

1. Viroids fulfill all structural criteria postulated for primitive replicons, including small size, $\mathrm{G}+\mathrm{C}$-rich content and compact folding, as well as circularity that may ensure complete replication and even polyploidy. 
2. From a functional perpective, viroids lack protein-coding ability but at least some "encode" ribozymes; in other words, they are catalytic RNAs, the key signature expected for survivors of the RNA World.

3. The three catalytic activities mediating rolling-circle replication of viroids, namely, RNA polymerization, cleavage and ligation, can be supplied by natural or artificial ribozymes, making reasonable replication in a protein-free world.

4. Viroid-like satellite RNAs, retroviroid-like elements and HDV RNA, can be regarded as evolutionary "inventions" related to (and possibly deriving from) viroids.

5. Feasible evolutionary routes can be envisaged for the transition of viroids and viroid-related replicons from the RNA World to a world with proteins and DNA.

Science runs through winding roads. The initial purpose of characterizing the viruses presumed to incite several plant diseases turned out eventually, and unexpectedly, in the discovery of a new class of subviral agents, the viroids. Their properties are consistent with those predicted for the first minimal replicons that populated the RNA World, bolstering the case for its existence.

\section{DISCLOSURE STATEMENT}

The authors are not aware of any affiliations, memberships, funding, or financial holdings that might be perceived as affecting the objectivity of this review.

\section{ACKNOWLEDGEMENTS}

Research in R.F. laboratory is funded by grant BFU2011-28443 from Ministerio de Economía y Competititvidad (MINECO, Spain), in R.S. laboratory by grants BFU2011-25271 from MINECO and the European Research Council (ERC-2011-StG281191-VIRMUT), and in S.F.E. laboratory by grant BFU2012-30805 from MINECO. P.S. has been supported by postdoctoral contracts from the Generalitat Valenciana (APOSTD/2010, program VALi+d) and MINECO (program Juan de la Cierva). 


\section{LITERATURE CITED}

1. Allison LA, Simon LD, Maliga P. 1996. Deletion of rpoB reveals a second distinct transcription system in plastids of higher plants. EMBO J. 15:28029

2. Ambrós S, Hernández C, Flores R. 1999. Rapid generation of genetic heterogeneity in progenies from individual cDNA clones of peach latent mosaic viroid in its natural host. J. Gen. Virol. 80:2239-52

3. Atkins JF, Gesteland RF, Cech TR. (Eds.) 2011. RNA Worlds: From Life's Origins to Diversity in Gene Regulation. Cold Spring Harbor: Cold Spring Harbor Laboratory Press, pp. 361

4. Beijerinck MW. 1898. Ueber ein Contagium vivum fluidum als Ursache der Fleckenkrankheit der Tabaksblatter. Verh. K. Akad. Wet. Amsterdam 65:3-21

5. Bernad L, Duran-Vila N, Elena SF. 2009. Effect of citrus hosts on the generation, maintenance and evolutionary fate of genetic variability of citrus exocortis viroid. J. Gen. Virol. 90:2040-9

6. Biebricher CK, Eigen M. 2006. What is a quasispecies?. Curr. Top. Microbiol. Immunol. 299:1-31

7. Branch AD, Robertson HD. 1984. A replication cycle for viroids and other small infectious RNAs. Science 223:450-4

8. Brazas, R., Ganem. D. 1996. A cellular homolog of hepatitis delta antigen: implications for viral replication and evolution. Science 274:90-4.

9. Briones C, Stich M, Manrubia SC. 2009. The dawn of the RNA World: toward functional complexity through ligation of random RNA oligomers. RNA 15:743-9

10. Bruening G, Passmore BK, van Tol H, Buzayan JM, Feldstein PA. 1991. Replication of a plant virus satellite RNA: evidence favors transcription of circular templates of both polarities. Mol. Plant Microbe Interact. 4:219-25

11. Burch CL, Chao L. 1999. Evolution by small steps and rugged landscapes in the RNA virus $\phi 6$. Genetics 151:921-7

12. Buzayan JM, Gerlach WL, Bruening, G. 1986. Non-enzymatic cleavage and ligation of RNAs complementary to a plant virus satellite RNA. Nature 323, 349-53

13. Cech TR. 2000. Structural biology. The ribosome is a ribozyme. Science 289:878-9 
14. Chela-Flores J. 1994. Are viroids molecular fossils of the RNA world? J. Theor. Biol. 166:163-6

15. Chen PJ, Kalpana G, Goldberg J, Mason W, Werner B, Gerin J, Taylor J. 1986. Structure and replication of the genome of the hepatitis $\partial$ virus. Proc. Natl. Acad. Sci. USA 83:8774-8

16. Codoñer FM, Daròs JA, Solé RV, Elena SF. 2006. The fittest versus the flattest: experimental confirmation of the quasispecies effect with subviral pathogens. PLoS Path. 2:1187-93

17. Cramer P, Armache KJ, Baumli S, Benkert S, Brueckner F, Buchen C, Damsma GE, Dengl S, Geiger SR, Jasiak AJ, Jawhari A, Jennebach S, Kamenski T, Kettenberger H, Kuhn CD, Lehmann E, Leike K, Sydow JF, Vannini A. 2008. Structure of eukaryotic RNA polymerases. Annu. Rev. Biophys. 37:337-52.

18. Crick FH. 1968. The origin of the genetic code. J. Mol. Biol. 38:367-79

19. Daròs JA, Marcos JF, Hernández C, Flores R. 1994. Replication of avocado sunblotch viroid: Evidence for a symmetric pathway with two rolling circles and hammerhead ribozyme processing. Proc. Natl. Acad. Sci. USA 91:12813-7

20. Daròs JA, Flores R. 1995. Identification of a retroviroid-like element from plants. Proc. Natl. Acad. Sci. USA 92:6856-60

21. Daròs JA, Flores R. 2002. A chloroplast protein binds a viroid RNA in vivo and facilitates its hammerhead-mediated self-cleavage. $E M B O J$ J 21:749-59

22. De la Peña M, Navarro B, Flores, R. 1999. Mapping the molecular determinant of pathogenicity in a hammerhead viroid: a tetraloop within the in vivo branched RNA conformation. Proc. Natl. Acad. Sci. USA 96:9960-5

23. De la Peña M, García-Robles I. 2010. Ubiquitous presence of the hammerhead ribozyme motif along the tree of life. RNA 16:1943-50

24. Delgado S, Martínez de Alba AE, Hernández C, Flores R. 2005. A short double-stranded RNA motif of peach latent mosaic viroid contains the initiation and the self-cleavage sites of both polarity strands. J. Virol. 79:1293443

25. den Boon JA, Ahlquist P. 2010. Organelle-like membrane compartmentalization of positive-strand RNA virus replication factories. Annu. Rev. Microbiol. 64: 241-56

26. Diener TO. 1971. Potato spindle tuber "virus": IV. A replicating, low molecular weight RNA. Virology 45:411-428 
27. Diener TO. 1972. Potato spindle tuber viroid VIII. Correlation of infectivity with a UV-absorbing component and thermal denaturation properties of the RNA. Virology 50:606-9

28. Diener TO. 1981. Are viroids escaped introns?. Proc. Natl. Acad. Sci. USA 78:5014-5

29. Diener TO. 1989. Circular RNAs: relics of precellular evolution? Proc. Natl. Acad. Sci. USA 86:9370-4

30. Diener TO. 2003. Discovering viroids: a personal perspective. Nat. Rev. Microbiol. 1:75-80

31. Ding B. 2009. The biology of viroid-host interactions. Annu. Rev. Phytopathol. 47:105-31

32. Ding B. 2010. Viroids: self-replicating, mobile, and fast-evolving noncoding regulatory RNAs. Wiley Interdiscip. Rev. RNA 1:362-75

33. Domingo E, Sabo D, Taniguchi T, Weissmann C, 1978. Nucleotide sequence heterogeneity of an RNA phage population. Cell 13:735-44

34. Eigen M. 1971. Self-organization of matter and the evolution of biological macromolecules. Naturwissenschaften 58:465-523

35. Eigen M, Schuster P. 1978. The hypercycle. A principle of natural selforganization. Part C: the realistic hypercycle. Naturwissenschaften 65:341-69

36. Elena SF, Dopazo J, Flores R, Diener TO, Moya A. 1991. Phylogeny of viroids viroidlike satellite RNAs, and the viroidlike domain of hepatitis $\mathrm{d}$ virus RNA. Proc. Natl. Acad. Sci. USA 88:5631-34

37. Elena SF, Dopazo J, de la Peña M, Flores R, Diener TO, Moya A. 2001. Phylogenetic analysis of viroid and viroid-like satellite RNAs from plants: a reassessment. J. Mol. Evol. 53:155-9

38. Epstein LM and Gall JG. 1987. Self-cleaving transcripts of satellite DNA from the newt. Cell 48:535-43

39. Fadda Z, Daròs JA, Fagoaga C, Flores R, Duran-Vila N. 2003. Eggplant latent viroid (ELVd): candidate type species for a new genus within family Avsunviroidae (hammerhead viroids). J. Virol. 77, 6528-32

40. Fedor MJ. 2000. Structure and function of the hairpin ribozyme. J. Mol. Biol. 297:269-91

41. Feix G, Pollet R, Weissmann C. 1968. Replication of viral RNA, XVI. Enzymatic synthesis of infectious virual RNA with noninfectious Q-beta minus strands as template. Proc. Natl. Acad. Sci. USA 59:145-52 
42. Flores R, Semancik JS. 1982. Properties of a cell-free system for synthesis of citrus exocortis viroid. Proc. Natl. Acad. Sci. USA 79:6285-8

43. Flores R, Hernández C, Martínez de Alba E, Daròs JA, Di Serio F. 2005. Viroids and viroid-host interactions. Annu. Rev. Phytopathol. 43:117-39

44. Flores R, Di Serio F, Navarro B, Duran-Vila N, Owens RA. 2011. Viroids and viroid diseases of plants. In Studies in Viral Ecology, vol I, Microbial and Botanical Host Systems, ed. CJ Hurst, 311-46. Hoboken: John Wiley \& Sons

45. Flores R, Serra P, Minoia S, Di Serio F, Navarro B. 2012. Viroids: from genotype to phenotype just relying on RNA sequence and structural motifs. Front. Microbiol. 3:article 217

46. Forterre P. 2005. The two ages of the RNA world, and the transition to the DNA world: a story of viruses and cells. Biochimie 87:793-803

47. Forster AC, Symons RH. 1987. Self-cleavage of plus and minus RNAs of a virusoid and a structural model for the active sites. Cell 49:211-20

48. Fraenkel-Conrat H. 1956. The role of the nucleic acid in the reconstitution of active tobacco mosaic virus. J. Am. Chem. Soc. 78:882-3

49. Freeland SJ, Knight RD, Landweber LF. 1999. Do proteins predate DNA? Science 286:690-2

50. Fresco JR, Alberts BM, Doty P. 1960. Some molecular details of the secondary structure of ribonucleic acid. Nature 188:98-101

51. Gago S, Elena SF, Flores R, Sanjuán R. 2009. Extremely high variability of a hammerhead viroid. Science 323:1308

52. Gandía M, Duran-Vila N. 2004. Variability of the progeny of a sequence variant of citrus bent leaf viroid (CBLVd). Arch. Virol. 149:407-16

53. Gas ME, Hernández C, Flores R, Daròs JA. 2007. Processing of nuclear viroids in vivo: an interplay between RNA conformations. PLoS Pathog. 3:181326

54. Gierer A, Schramm G. (1956). Infectivity of ribonucleic acid from tobacco mosaic virus. Nature 177:702-3

55. Gilbert W. 1986. Origin of life: the RNA world. Nature 319:618

56. Góra-Sochacka A, Kierzek A, Candresse T. Zagórski W. 1997. The genetic stability of potato spindle tuber viroid (PSTVd) molecular variants. RNA 3:6874 
57. Grill LK, Semancik JS. 1978. RNA sequences complementary to citrus exocortis viroid in nucleic acid preparations from infected Gynura aurantiaca. Proc. Natl. Acad. Sci. USA 75:896-900

58. Gross HJ, Domdey H, Lossow C, Jank P, Raba M, Alberty H, Sänger HL. 1978. Nucleotide sequence and secondary structure of potato spindle tuber viroid. Nature 273:203-8

59. Gudima S, Wu SY, Chiang CM, Moraleda G, Taylor J. 2000. Origin of hepatitis $\delta$ virus mRNA. J. Virol. 74:7204-10

60. Guerrier-Takada C, Gardiner K, Marsh T, Pace N, Altman S. 1983. The RNA moiety of ribonuclease $\mathrm{P}$ is the catalytic subunit of the enzyme. Cell 35:849-57

61. Haag JR, Pikaard CS. 2011. Multisubunit RNA polymerases IV and V: purveyors of non-coding RNA for plant gene silencing. Nat. Rev. Mol. Cell. Biol. 12: 483-92

62. Hadidi A. 1986. Relationship of viroids and certain other plant pathogenic nucleic acids to group I and II introns. Plant Mol. Biol. 7:129-42

63. Hajeri S, Ramadugu C, Manjunath K, Ng J., Lee R, Vidalakis G. 2011. In vivo generated citrus exocortis viroid progeny variants display a range of phenotypes with altered levels of replication, systemic accumulation and pathogenicity. Virology 417:400-9

64. Hammann C, Luptak A, Perreault J, de la Peña M. 2012. The ubiquitous hammerhead ribozyme. RNA 18:871-85

65. Hammann C, Steger G. 2012. Viroid-specific small RNA in plant disease. RNA Biol. 9:809-19

66. Hammond R, Smith DR, Diener TO. 1989. Nucleotide sequence and proposed secondary structure of Columnea latent viroid: a natural mosaic of viroid sequences. Nucleic Acids Res. 17:10083-94

67. Haruna I, Spiegelman S. 1966. Proc. Natl. Acad. Sci. USA 55:1256-63

68. Hegedus K, Dallmann G, Balazs E. 2004. The DNA form of a retroviroidlike element is involved in recombination events with itself and with the plant genome. Virology 325: 277-86

69. Hernández C, Flores R. 1992. Plus and minus RNAs of peach latent mosaic viroid self-cleave in vitro via hammerhead structures. Proc. Natl. Acad. Sci. USA 89:3711-5 
70. Hernández C, Daròs, JA, Elena, SF, Moya A, Flores R. 1992. The strands of both polarities of a small circular RNA from carnation self-cleave in vitro through alternative double- and single hammerhead structures. Nucleic Acids Res. 20:6323-9

71. Ho CK, Shuman S. 2002. Bacteriophage T4 RNA ligase 2 (gp24.1) exemplifies a family of RNA ligases found in all phylogenetic domains. Proc. Natl. Acad. Sci. USA 99: 12709-14

72. Hutchins CJ, Keese P, Visvader JE, Rathjen PD, McInnes JL, Symons RH. 1985. Comparison of multimeric plus and minus forms of viroids and virusoids. Plant Mol. Biol. 4:293-304

73. Hutchins C, Rathjen PD, Forster AC, Symons RH. 1986. Self-cleavage of plus and minus RNA transcripts of avocado sunblotch viroid. Nucleic Acids Res. 14:3627-40

74. Ivica NA, Obermayer B, Campbell GW, Rajamani S, Gerland U, Chen IA. 2013. The paradox of dual roles in the RNA world: resolving the conflict between stable folding and templating ability. J. Mol. Evol. 77:55-63

75. Jenkins GM, Woelk CH, Rambaut A, Homes EC. 2000. Testing the extent of sequence similarity among viroids, satellite RNAs, and the hepatitis delta virus. J. Mol. Evol. 50:98-102

76. Johnston WK, Unrau PJ, Lawrence MS, Glasner ME, Bartel DP. 2001. RNAcatalyzed RNA polymerization: accurate and general RNA-templated primer extension. Science 292:1319-25

77. Juhasz A, Hegyi H, Solymosy F. 1988. A novel aspect of the information content of viroids. Biochim. Biophys. Acta 950:455-8

78. Kiefer MC, Owens RA, Diener TO. 1983. Structural similarities between viroids and transposable genetic elements. Proc. Natl. Acad. Sci. USA 80:6234-8

79. Kolonko N, Bannach O, Aschermann K, Hu KH, Moors M, Schmitz M, Steger G, Riesner D. 2006. Transcription of potato spindle tuber viroid by RNA polymerase II starts in the left terminal loop. Virology 347:392-404

80. Kruger K, Grabowski PJ, Zaug AJ, Sands J, Gottschling DE, Cech TR. 1982. Self-splicing RNA: autoexcision and autocyclization of the ribosomal RNA intervening sequence of Tetrahymena. Cell 31:147-57

81. Kuo MY, Goldberg J, Coates L, Mason W, Gerin J, Taylor J. 1988a. Molecular cloning of hepatitis delta virus RNA from an infected woodchuck liver: sequence, structure, and applications. J. Virol. 62:1855-61 
82. Kuo MY, Sharmeen L, Dinter-Gottlieb G, Taylor J. 1988b. Characterization of self-cleaving RNA sequences on the genome and antigenome of human hepatitis delta virus. J. Virol. 62:4439-44

83. Lauring AS, Andino R. 2010. Quasispecies theory and the behavior of RNA viruses. PLoS Pathog. 6: e1001005

84. Lazcano A, Guerrero R, Margulis L, Oro J. 1988. The evolutionary transition from RNA to DNA in early cells. J. Mol. Evol. 27: 283-90

85. Lima MI, Fonseca MEN, Flores R, Kitajima EW. 1994. Detection of avocado sunblotch viroid in chloroplasts of avocado leaves by in situ hybridization. Arch. Virol. 138:385-90

86. Lincoln TA, Joyce GF. 2009. Self-sustained replication of an RNA enzyme. Science 323:1229-32

87. Manrubia SC, Briones C. 2007. Modular evolution and increase of functional complexity in replicating RNA molecules. RNA 13:97-107

88. Margulis L. 1993. Symbiosis in cell evolution, 2nd ed. W. H. Freeman and Co., New York, N.Y., pp. 452

89. McInnes JL, Symons RH. 1991. Comparative structure of viroids and their rapid detection using radioactive and nonradioactive nucleic acid probes. In Viroids and Satellites: Molecular Parasites at the Frontier of Life, ed. K Maramorosch, pp. 21-58, Boca Raton: CRC Pres

90. Moelling K. 2013. What contemporary viruses tell us about evolution: a personal view. Arch. Virol. 158:1833-48

91. Mühlbach HP, Sänger HL. 1979. Viroid replication is inhibited by $\alpha$ amanitin. Nature 278:185-8

92. Navarro B, Flores R. 1997. Chrysanthemum chlorotic mottle viroid: unusual structural properties of a subgroup of viroids with hammerhead ribozymes. Proc. Natl. Acad. Sci. USA 94:11262-7

93. Navarro JA, Flores R. 2000. Characterization of the initiation sites of both polarity strands of a viroid RNA reveals a motif conserved in sequence and structure. EMBO J 19:2662-70

94. Navarro JA, Vera A, Flores R. 2000. A chloroplastic RNA polymerase resistant to tagetitoxin is involved in replication of avocado sunblotch viroid. Virology 268:218-25 
95. Navarro B, Gisel A, Rodio ME, Delgado S, Flores R, Di Serio F. 2012a. Viroids: how to infect a host and cause disease without encoding proteins. Biochimie 94:1474-80

96. Navarro B, Gisel A, Rodio ME, Delgado S, Flores R, Di Serio F. 2012b. Small RNAs containing the pathogenic determinant of a chloroplastreplicating viroid guide the degradation of a host mRNA as predicted by RNA silencing. Plant J. 70:991-1003

97. Nohales MA, Molina-Serrano D, Flores R, Daròs JA. 2012a. Involvement of the chloroplastic isoform of tRNA ligase in the replication of the viroids belonging to the family Avsunviroidae. J. Virol. 86: 8269-76

98. Nohales MA, Flores R, Daròs JA. 2012b. A viroid RNA redirects host DNA ligase 1 to act as an RNA ligase. Proc. Natl. Acad. Sci. USA 109:13805-10

99. Ojosnegros S, Perales C, Mas A, Domingo E. 2011. Quasispecies as a matter of fact: viruses and beyond. Virus Res. 162:203-15

100. Orgel LE. 1968. Evolution of the genetic apparatus. J. Mol. Biol. 38:381-93

101. Pascal JM. 2008. DNA and RNA ligases: Structural variations and shared mechanisms. Curr. Opin. Struct. Biol. 18:96-105

102. Pascal JM, O'Brien PJ, Tomkinson AE, Ellenberger T. 2004. Human DNA ligase I completely encircles and partially unwinds nicked DNA. Nature 432:473-8

103. Poole AM, Logan DT. 2005. Modern mRNA proofreading and repair: clues that the last universal common ancestor possessed and RNA genome? Mol. Biol. Evol. 22:1444-55

104. Powner MW, Gerland B, Sutherland JD. 2009. Synthesis of activated pyrimidine ribonucleotides in prebiotically plausible conditions. Nature 459:239-42

105. Prody GA, Bakos JT, Buzayan JM, Schneider IR, Bruening G. 1986. Autolytic processing of dimeric plant virus satellite RNA. Science 231:1577-80

106. Reanney D. 1984. Genetic noise in evolution? Nature 307:318-9

107. Rezaian MA. 1990. Australian grapevine viroid-evidence for extensive recombination between viroids. Nucleic Acids Res. 18:1813-8.

108. Riesner D, Henco K, Rokohl U, Klotz G, Kleinschmidt AK, Domdey H, Jank P, Gross HJ, Sänger HL. 1979. Structure and structure formation of viroids. J. Mol.Biol. 133: 85-115 
109. Rizzeto M, Canese MG, Aricò J, Crivelli O, Trepo C, Bonino F, Verme G. 1977. Immunofluorescence detection of a new antigen-antibody system associated to the hepatitis B virus in the liver and in the serum of HBsAg carriers. Gut 18:997-1003

110. Rizzetto M, Hoyer B, Canese MG, Shih JW, Purcell RH, Gerin JL. 1980. $\partial$ agent: Association of $\partial$ antigen with hepatitis B surface antigen and RNA in serum of $\partial$-infected chimpanzees. Proc. Natl. Acad. Sci. USA 77:6124-8

111. Robertson HD. 1996. How did replicating and coding RNAs first get together? Science 274:66-7

112. Rodio ME, Delgado S, De Stradis AE, Gómez MD, Flores R, Di Serio, F. 2007. A viroid RNA with a specific structural motif inhibits chloroplast development. Plant Cell 19:3610-26

113. Roossinck MJ, Sleat D, Palukaitis P. 1992. Satellite RNAs of plant viruses: structures and biological effects. Microbiol. Rev. 56: 265-79

114. Sanjuán R, Forment J, Elena SF. 2006a. In silico predicted robustness of viroids RNA secondary structures. I. The effect of single mutations. Mol. Biol. Evol. 23:1427-36

115. Sanjuán R, Forment J, Elena SF. 2006b. In silico predicted robustness of viroid RNA secondary structures. II. Interaction between mutation pairs. Mol. Biol. Evol. 23:2123-30

116. Sanjuán R, Cuevas JM, Furió V, Holmes EC, Moya A. 2007. Selection for robustness in mutagenized RNA viruses. PLoS Genet. 3:939-46

117. Schiebel W, Pélissier T, Riedel L, Thalmeir S, Schiebel R, Kempe D, Lottspeich F, Sänger HL, Wassenegger M. 1998. Isolation of an RNA-directed RNA polymerase-specific cDNA clone from tomato. Plant Cell 10:2087-101

118. Schindler IM, Mühlbach HP. 1992. Involvement of nuclear DNAdependent RNA polymerases in potato spindle tuber viroid replication: a reevaluation. Plant Sci. 84:221-9

119. Schneider R. 1969. Satellite-like particle of tobacco ringspot virus that resembles tobacco ringspot virus. Science 166:1627-9

120. Schuster P, Swetina J. 1988. Stationary mutant distributions and evolutionary optimization. Bull. Math. Biol. 50:635-60

121. Semancik JS, Szychowski JA, Rakowski AG, Symons RH. 1993. Isolates of citrus exocortis viroid recovered by host and tissue selection. J. Gen. Virol. 74:2427-36 
122. Shuman S, Lima CD. 2004. The polynucleotide ligase and RNA capping enzyme superfamily of covalent nucleotidyltransferases. Curr. Opin. Struct. Biol. 14:757-64

123. Sogo JM, Koller T, Diener TO. 1973. Potato spindle tuber viroid X. Visualization and size determination by electron microscopy. Virology 55:70-80 124. Sugiura M. 1992. The chloroplast genome. Plant Mol. Biol. 19:149-68

125. Symons RH. 1981. Avocado sunblotch viroid: Primary sequence and proposed secondary structure. Nucleic Acids Res. 9:6527-37

126. Symons RH, Randles JW. 1999. Encapsidated circular viroidlike satellite RNAs (virusoids) of plants. Curr. Top. Microbiol. Immunol. 239:81-105

127. Tabler M, Tsagris M. 2004. Viroids: petite RNA pathogens with distinguished talents. Trends Plant Sci. 9:339-48

128. Taylor JM. 2009. Replication of the hepatitis delta virus RNA genome. Adv. Virus Res. 74:103-21

129. Taylor J, Pelchat M. 2010. Origin of hepatitis delta virus. Future Microbiol. 5:393-402

130. Tessitori M, Rizza S, Reina A, Causarano G, Di Serio F. 2013. The genetic diversity of Citrus dwarfing viroid populations is mainly dependent on the infected host species. J. Gen. Virol. 94:687-93

131. Tsagris EM, Martínez de Alba AE, Gozmanova M, Kalantidis K. 2008. Viroids. Cell Microbiol. 10:2168-79

132. Verhoeven JT, Meekes ET, Roenhorst JW, Flores R, Serra P. 2013. Dahlia latent viroid: a recombinant new species of the family Pospiviroidae posing intriguing questions about its origin and classification. J. Gen. Virol. 94:711-9

133. Vignuzzi M, Stone JK, Arnold JJ, Cameron CE, Andino R. 2006. Quasispecies diversity determines pathogenesis through cooperative interactions in a viral population. Nature 439:344-8

134. Visvader JE, Symons RH. 1985. Eleven new sequence variants of citrus exocortis viroid and the correlation of sequence with pathogenicity. Nucleic Acids Res. 13:2907-20

135. Wang KS, Choo QL, Weiner AJ, Ou JH, Najarian RC, Thayer RM, Mullenbach GT, Denniston KJ, Gerin JL, Houghton M. 1986. Structure, sequence and expression of the hepatitis delta ( $\partial$ ) viral genome. Nature 323:508-14 (Authors' correction, 328:456) 
136. Wang LK, Schwer B, Shuman S. 2006. Structure-guided mutational analysis of T4 RNA ligase 1. RNA 12:2126-34

137. Wassenegger M, Spieker RL, Thalmeir S, Gast FU, Riedel L, Sänger HL. 1996. A single nucleotide substitution converts potato spindle tuber viroid (PSTVd) from a noninfectious to an infectious RNA for Nicotiana tabacum. Virology 226:191-7.

138. Wassenegger M, Krczal G. 2006. Nomenclature and functions of RNAdirected RNA polymerases. Trends Plant Sci. 11:142-51

139. Webb C-HT, Riccitelli NJ, Ruminski DJ, Lupták A. 2009. Widespread occurrence of self-cleaving ribozymes. Science 326:953

140. Wilke CO, Wang JL, Ofria C, Lenski RE, Adami C. 2001. Evolution of digital organisms at high mutation rates leads to survival of the flattest. Nature 412:331-3

141. Wochner A, Attwater J, Coulson A, Holliger P. 2011. Ribozyme-catalyzed transcription of an active ribozyme. Science 332:209-12

142. Woese CR. 1967. The genetic code: the molecular basis for genetic expression. New York: Harper and Row, pp 200

143. Wu Q, Wang Y, Cao M, Pantaleo V, Burgyan J, Li WX, Ding SW. 2012. Homology-independent discovery of replicating pathogenic circular RNAs by deep sequencing and a new computational algorithm. Proc. Natl. Acad. Sci. USA 109:3938-43

144. Xiong Y, Eickbush TH. 1990. Origin and evolution of retroelements based upon their reverse transcriptase sequences. EMBO J. 9:3353-62

145. Zhang ZX, Chen SS, Qi SS, Ding SW, Wu QF, Li. SF. 2013. Identification of new viroid-like circular RNAs from grapevine and apple plants. Internatl. Workshop on Viroids and Satellite RNAs, p. 19, Beijing, China

146. Zhong X, Archual AJ, Amin AA, Ding B. 2008. A genomic map of viroid RNA motifs critical for replication and systemic trafficking. Plant Cell 20:35-47. 


\section{Legends to Figures}

\section{Figure 1.}

Viroid structure. Top, rod-like secondary structure with a central conserved region (CCR) proposed for PSTVd (family Pospiviroidae. Bottom, branched secondary structures proposed for PLMVd (family Avsunviroidae) (left) and for its plus polarity hammerhead ribozyme (right). Nucleotides strictly or highly conserved in natural hammerhead structures are shown within boxes with blue and white backgrounds for plus and minus polarities, respectively. Continuous lines and dots between nucleotides represent canonical (Watson-Crick) and non-canonical base pairs, respectively, with the arrow marking the self-cleavage site. Broken rectangles denote tertiary interactions between loops that either stabilize the global viroid conformation or promote the catalytically-active ribozyme folding.

\section{Figure 2.}

Viroid classification. Distinctive structural and functional features of the two viroid families.

\section{Figure 3.}

Asymmetric and symmetric variants of the rolling-circle mechanism proposed for replication of members of the families Pospiviroidae and Avsunviroidae, respectively. Yellow and blue colors refer to plus and minus polarities, respectively, with cleavage sites denoted by arrowheads. The enzymes and ribozymes that presumably catalyze the replication steps are indicated. Notice that RNA polymerase II is redirected to transcribe RNA templates and DNA ligase I to circularize RNA substrates. NEP is an abbreviation for nuclear encoded polymerase and HHRz for hammerhead ribozyme.

\section{Figure 4.}

Characteristics of viroids supporting that they may have a very old origin.

\section{Figure 5.}

Per-site mutation rate versus genome size for CChMVd and other biological entities. RNA viruses (left to right) are Tobacco mosaic virus, human rhinovirus, poliovirus, Vesicular stomatitis virus, bacteriophage $\phi 6$, and Measles virus. Single-stranded DNA viruses are bacteriophage $\phi X 174$ and bacteriophage m13. Double-stranded DNA 
viruses are bacteriophage 1 , Herpes simplex virus, bacteriophage $\mathrm{T} 2$, and bacteriophage T4. Bacteria is Escherichia coli. Lower eukaryotes are Saccharomyces cerevisiae and Neurospora crassa. Higher eukaryotes are Caenorhabditis elegans, Drosophila melanogaster, Mus musculus, and Homo sapiens. When several estimations were available, the mean value is shown. (Reproduced with permission from Gago et al., Science 2009) 